\title{
Psychometric Evaluation of the German Version of the Dietary Fat and Free Sugar-Short Questionnaire
}

\author{
Sophie Pauline Fromm ${ }^{a}$ b Annette Horstmann ${ }^{a, c, d}$ \\ aDepartment of Neurology, Max Planck Institute for Human Cognitive and Brain Sciences,

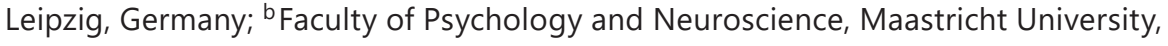 \\ Maastricht, The Netherlands; 'IFB AdiposityDiseases, Leipzig University, Leipzig, Germany; \\ ${ }^{\mathrm{d}}$ Department of Psychology and Logopedics, Faculty of Medicine, University of Helsinki, \\ Helsinki, Finland
}

\section{Keywords}

Nutritional assessment . Short questionnaire $\cdot$ Diet $\cdot$ Saturated fat $\cdot$ Free sugars

\begin{abstract}
Background: The Dietary Fat and Free Sugar-Short Questionnaire (DFS) is a cost- and timeefficient self-report screening instrument to estimate dietary intake of saturated fat and free sugar. To date, only the English version has been psychometrically evaluated. We assessed the psychometric characteristics of the German version of the DFS in individuals with normal weight, overweight, and obesity. Method: Sixty-five adult participants completed a German translation of the DFS and a validated food frequency questionnaire (FFQ). We correlated participants' percentage of energy intake from saturated fat and free sugar from the FFQ with the DFS scores. To establish test-retest reliability, participants completed the DFS a second time. To investigate convergent validity, we correlated participants' DFS scores with self-reported eating behavior and sensitivity to reward. Results: DFS scores correlated with percentage of energy from free sugar ( $r s=0.443$ ) and saturated fatty acids $(r s=0.258)$ but not with non-target nutrients. The correlation between DFS scores and percentage energy from free sugar was not moderated by body mass index (BMI), whereas the correlation with percentage energy from saturated fat slightly decreased with BMI. Intra-class correlation as an indicator of test-retest reliability was 0.801 . DFS scores correlated significantly with restraint of eating behavior $(r s=-0.380$ ) and feelings of hunger $(r s=0.275)$. Correlations of the DFS score with disinhibited eating and sensitivity to rewards failed to be significant. Conclusion: Our data indicate that the German version of the DFS might be a psychometrically sound self-report instrument to estimate saturated fat and free sugar intake of German adults.
\end{abstract}




\section{Introduction}

The four major risk factors for non-communicable diseases identified as overweight, raised blood pressure, and increased lipid and glucose blood levels are suggested to depend largely on nutrition [1]. As such, the improvement of dietary behavior to aid overweight and associated health adversities is a major health concern and in the focus of present prevention and treatment programs. Two potential candidates for dietary interventions are saturated fat and free sugar. Free sugar is defined as all monosaccharides and disaccharides added to foods and beverages by the manufacturer, cook, or consumer, and sugars naturally present in honey, syrups, fruit juices, and concentrates [2]. High free sugar intake was associated with impaired glucose tolerance and insulin resistance as well as increased serum cholesterol and triglycerides that all seem to increase the risk for myocardial infarction and cardiovascular disease [3]. Moreover, consumption of free sugar and sugar-sweetened beverages has been associated with higher risk to develop a variety of health adversities that are typical for the metabolic syndrome, such as type 2 diabetes [4], increased blood pressure [5], and weight gain [6-8]. High dietary saturated fat intake appears to have unfavorable effects on blood lipid profiles because it raises low-density lipoprotein levels which eventually increases the risk for cardiovascular diseases $[9,10]$. Taken together, the intake of saturated fat and free sugar contributes to the rise of non-communicable diseases and obesity and should be limited. The leading German societies for obesity, diabetes, and nutrition recommend limiting free sugar and saturated fat intake, so that the percentage of energy from saturated fat and free sugar does each not exceed $10 \%$ of total energy intake $[11,12]$.

To estimate the proportion of free sugar and saturated fat in a diet, adequate and valid assessment instruments, such as the Dietary Fat and free Sugar-Short Questionnaire (DFS) [13], are indispensable. The aim of this study was to investigate the psychometric properties of the German version of the DFS and its relative validity against a more comprehensive food frequency questionnaire (FFQ). Specifically, we focus on the question whether the DFS is equally valid for people with a body mass index (BMI) in the range of normal weight, overweight, and obesity. To provide empirical evidence for the convergent validity of the German version of the DFS, we assessed if research that relates high free sugar and saturated fat intake to certain eating behavior characteristics can be replicated with the DFS. High intake of saturated fat and free sugar appears to be associated with less restraint [14], more disinhibition [15], and feelings of hunger [16] as well as a higher sensitivity to reward [17, 18]. Besides certain eating behavior characteristics, high free sugar and saturated fat intake is reported to be more prevalent in the male and the younger population.

Screening tools and short dietary questionnaires are indispensable to assess dietary habits and nutrient intake in clinical practice in a structured and standardized way. Various German assessment tools, including FFQs, dietary records, and fat screening tools are available [19-21]. However, none of these simultaneously assesses the intake of saturated fat and free sugar in a cost- and time-saving way. With the German version of the DFS, it may become possible to assess dietary habits with respect to saturated fat and free sugar intake more conveniently.

\section{Method}

Participants and Procedures

Participants were extracted from the database of the Max Planck Institute for Human Cognitive and Brain Science (MPI/CBS) and contacted via telephone. This study was conducted according to the standards laid down in the Declaration of Helsinki. All procedures were 
approved by the MPI/CBS Ethics Committee. After receiving basic study information and providing written informed consent on the first appointment, 72 individuals took part in the study. Five participants were excluded due to the presence of depressive symptoms or disordered eating behavior as indicated by self-report. Two participants did not take part in the second assessment at follow-up. Therefore, 65 participants were included in the analysis. The sample was selected to include a similar number of individuals with normal weight (BMI $<25$ $\mathrm{kg} / \mathrm{m}^{2}$ ), overweight (BMI $25-29.9 \mathrm{~kg} / \mathrm{m}^{2}$ ), and obesity (BMI $\geq 30 \mathrm{~kg} / \mathrm{m}^{2}$ ).

Participants were screened on the telephone for the following exclusion criteria: (1) regular consumption of nicotine within the last year; (2) alcohol consumption $>20 \mathrm{~g} /$ day; (3) history of drug abuse; (4) current pregnancy or breastfeeding; (5) head injury that led to loss of consciousness; (6) diabetes mellitus or thyroid malfunction; (7) past or current neurological or psychiatric disorders; (8) intention to diet in the past or upcoming 3 weeks. By means of self-report measures, participants with (9) increased depressive symptoms and (10) disordered eating were excluded from the analyses, but not from study participation. We excluded participants with depressive symptoms as indicated by a score $>18$ on the Beck Depression Inventory (BDI) [22] because we assumed depression to potentially impact the eating behavior and thus, valid reporting thereof. Participants with disordered eating were excluded from the analysis because they might potentially have a distorted view on their own food intake that could affect their ratings. As the total score of the EDE-Q varies with BMI [23, $24]$, cut-offs were calculated according to individual BMI based on previously reported norms $[23,24]$.

After providing written informed consent, participants' height and weight were measured. The BDI was administered on paper and evaluated immediately to register suicidal participants and refer them to a study physician. Otherwise, participants were guided to the computer laboratory to complete the remaining self-reports implemented in LimeSurvey software [25]. Participants were randomized to either complete first the DFS or the comprehensive FFQ. As a next step, they completed the Three-Factor Eating Questionnaire (TFEQ) [26], the Behavioral Inhibition System/Behavioral Activation System Scales (BIS/BAS Scales) [27], and the Eating Disorder Examination-Questionnaire (EDE-Q) [28] in fixed order. After being debriefed, participants were financially reimbursed with EUR 9 per hour for taking part in the study. At follow-up 2 weeks after the first appointment, participants were asked to follow a link to the DFS and complete it a second time within 14-25 days after the first appointment. If participants completed the follow-up test, they received additional reimbursement of EUR 4.50.

\section{Materials}

Saturated Fat and Free Sugar Intake

The DFS is a self-report instrument to assess the frequency of dietary intake of food rich in saturated fat and free sugar. It is structured like a checklist and consists of 26 items: 24 items ask respondents to recall the frequency of consumption of several food groups eaten in the last 12 months; 2 more items concern the frequency eaten away from home and the addition of sugar to beverages or food. Responses are given on a scale from 1 to 5 , with 1 reflecting having eaten the food "less than once per month" and 5 reflecting having eaten it " $5+$ per week." The last item concerns the number of teaspoons added to beverages, cereals, or food per week and is rated on a scale from 1 to 5 , with 1 reflecting "none" and 5 reflecting "7+" teaspoons. The total DFS score can range from 26 to 130 points. The DFS comprises three subscales, one reflecting food items that contribute only saturated fat (items 1-12), one contributing only free sugar (items 17, 20, 21, 23, 24, 26) and one contributing saturated fat and free sugar (remaining items). With these scales, participants that eat a lot of free sugar or saturated fat only could be examined separately. Convergent and divergent validity of the 
subscales was examined using a dietary diary and FFQ. Correlations of the diary and FFQ with the saturated fat scale ranged between 0.33 and 0.6 , with the free sugar scale between 0.35 and 0.59 , and with the fat-sugar scale between 0.29 and 0.68 . To calculate the total DFS score, subscale scores are summed up. Internal consistency of the total score was $\alpha=0.76$ [13]. Completion of the DFS took approximately $5 \mathrm{~min}$. The translation of the English version of the DFS into German language was carried out in a collaborative and iterative manner by affiliates of the MPI/CBS who have lived in English-speaking countries for several years. Translation was completed before study start. The German DFS version is reported in the additional material.

Total Dietary Intake

We estimated nutrient intake by means of the DEGS-Ernährungsfragebogen [19]. This FFQ records the frequency (how often) and amount (how much) of 53 foods and beverages consumed in the last 4 weeks. For a more accurate and comparable estimation of the portion size, pictures of the inquired foods are shown next to the items. The DEGS-Ernährungsfragebogen is a psychometrically sound and established FFQ that showed good relative validity compared to 24-h dietary recalls [19].

\section{Reward Sensitivity}

We assessed reward sensitivity by means of the German version of the Behavioural Inhibition System/Behavioural Ativation System Scales (BIS/BAS Scales) [27, 29]. Specifically, the BAS scale and the BAS Reward Responsiveness scale (BAS-RR) from the German version of the BIS/BAS Scales were used. Internal consistency of the BAS scale was estimated at $\alpha=$ 0.79 and of BAS-RR at $\alpha=0.57$ in a German sample $(n=1,881)[30]$.

Eating Behavior

The German version of the TFEQ [26, 31] was used to assess three dimensions of eating behavior: cognitive restraint, disinhibition, and feelings of hunger. Internal consistency of the German subscales was estimated at $\alpha=0.74-0.87$ [32]. The Eating Disorder ExaminationQuestionnaire (EDE-Q) [28, 33] is a self-report instrument to examine eating disorder symptoms that participants experienced within the past 4 weeks. We used the total score of the German version of the EDE-Q for exclusion purposes only. Internal consistency of the total score was estimated at $\alpha=0.97$ [33].

Depressive Symptoms

The German version of BDI [22,34] was used to measure the severity of depressive symptoms experienced within the past week. This questionnaire was only used for exclusion purposes.

Anthropometrics

Height and weight of the participants were measured in light clothing and without shoes to the nearest centimeter by means of a digital scale (Robusta 813, Seca) and a wall-mounted chart. Normal weight was defined as a BMI $<25 \mathrm{~kg} / \mathrm{m}^{2}$, overweight as a BMI $25-29.9 \mathrm{~kg} / \mathrm{m}^{2}$, and obesity as a BMI $\geq 30 \mathrm{~kg}$.

\section{Analysis}

The statistical analysis was carried out with IBM SPSS Statistics 21 and Microsoft Excel 2016. Using Prodi ${ }^{\circledR} 6$ Software based on the NutriGuide ${ }^{\circledR}$ (Nutri-Science GMBH), nutrient content of food and beverages that were listed in the FFQ was provided. Based on this information, nutrient intake per month was estimated for every subject. Nutrient intakes were 
Fromm and Horstmann: German Dietary Fat and Free Sugar-Short Questionnaire

transformed into percentage kilojoules $(\mathrm{kJ})$ of total $\mathrm{kJ}$ intake per month to examine nutrient composition instead of total intake. We decided to examine nutrient composition because total intake is highly individual, depends on body composition and activity level, and recommendations for nutrient intake are usually given as a proportion to total intake. To estimate percentage of intrinsic and free sugar in the food products, items of the FFQ were split into two groups. One group comprised food containing predominantly free sugar. The other group was formed by food with mainly intrinsic sugar, meaning sugar that was not added by the consumer or manufacturer. To investigate the association of the DFS scores and free sugar intake estimated with the FFQ, only the food group containing predominantly free sugar was used in the analysis. Free sugar occurs mostly in the form of sucrose [35]. Therefore, besides free sugar, also sucrose content of the items in the free sugar group was considered as indicator for free sugar intake. Although the DFS only assesses frequency of consumption, we decided to examine its reliability relative to the FFQ scores derived from reported frequency and amount of dietary intake. By using both pieces of information, we aimed to achieve a more precise estimation of the actual intake.

The intraclass correlation coefficient was calculated in a two-way fixed effects model as measure for test-retest reliability and Cronbach's $\alpha$ coefficient as measure for internal consistency. Given that DFS scores within gender groups were distributed normally and homogeneity of variances was confirmed, we used paired $t$ tests to compare the DFS scores between genders. We examined correlations of total DFS score with age, BMI, and estimated saturated fat and free sugar from the FFQ. Because nutrient scores, BMI, and age were not normally distributed, we used Spearman correlations. Correlations between the DFS and non-target nutrients (protein, mono- and polyunsaturated fatty acids, intrinsic sugar) were carried out to determine discriminant validity. In line with the approach of Francis and Stevenson [13], separate scores for food items rich in saturated fat, free sugar, or both nutrients were created and distinct correlations of these subscales with nutrient intake were calculated. To test whether the DFS score estimates the nutrient intake of individuals with normal weight, overweight, and obesity equally well, we conducted regression analyses and evaluated if the relationship between DFS scores and nutrient estimates is moderated by BMI. To evaluate the prediction of nutrient estimates by DFS scores at meaningful BMI values, we centered BMI and DFS scores prior to regression analyses. Using the upper and lower quartiles of the DFS score, we compared high and low DFS scorers regarding nutrient intake by means of twotailed Mann-Whitney U tests. Lastly, we investigated whether correlations between DFS scores and self-reported restraint, disinhibition, feelings of hunger, and sensitivity of reward on the BAS and BAS-RR scales are in line with previous research as stated above.

\section{Results}

The sample ( $n=65 ; 33$ women) consisted of normal-weight ( $n=23)$, overweight $(n=18)$, and obese $(n=24)$ participants $\left(\right.$ mean $_{\text {BMI }}=29.11$, BMI range: $\left.18.8-47.7 \mathrm{~kg} / \mathrm{m}^{2}\right)$ aged $18-35$ years $\left(\right.$ mean $\left._{\text {age }}=28.12\right)$. The DFS score ranged from 32 to 91 at first measurement $(n=65$, mean $=53.62, \mathrm{SD}=10.29)$ and from 31 to 74 at follow-up (mean $=54.52, \mathrm{SD}=9.39)$. There were no missing data. The test-retest reliability was $0.801,95 \%$ CI $0.694-0.873$. Cronbach's $\alpha$ as measure for internal consistency was $\alpha=0.808$. Internal consistency of the fat scale was $\alpha=0.666$, of the sugar scale $\alpha=0.513$, and of the fat-sugar scale $\alpha=0.703$. Intercorrelations of the total DFS score with the fat $(r=0.787)$, sugar $(r=0.696)$, and the fat-sugar subscale $(r=0.839)$, as well as intercorrelations between the subscales $(r=0.391-0.5)$ were significant $(p \leq 0.001)$. Mean DFS score and nutrient intake of women and men are shown in Table 1. 
Fromm and Horstmann: German Dietary Fat and Free Sugar-Short Questionnaire

Table 1. Energy intake and percentage of energy from saturated fat, free sugar, and free sucrose of men and women estimated with the DEGS-Ernährungsfragebogen (FFQ)

\begin{tabular}{|c|c|c|c|c|}
\hline & \multicolumn{2}{|c|}{$\operatorname{Men}(n=32)$} & \multicolumn{2}{|c|}{ Women $(n=33)$} \\
\hline & mean & SD & mean & SD \\
\hline Total energy, kJ & 235,986 & 78,169 & 220,795 & 124,633 \\
\hline Saturated fat & $16.04 \%$ & $3.48 \%$ & $16.17 \%$ & $3.18 \%$ \\
\hline Free carbohydrates & $16.21 \%$ & $6.86 \%$ & $19.89 \%$ & $8.91 \%$ \\
\hline Free sucrose & $7.38 \%$ & $3.40 \%$ & $9.57 \%$ & $3.84 \%$ \\
\hline
\end{tabular}

Table 2. Correlation (Spearman) of the DFS score and subscales with nutrient estimates from the FFQ

\begin{tabular}{llllll}
\hline & $\begin{array}{l}\text { Total energy } \\
\text { intake }\end{array}$ & Fat & Saturated fat & Free sugar & Free sucrose \\
& $0.603^{* *}$ & 0.192 & $0.258^{*}$ & $0.443^{* *}$ & $0.445^{* *}$ \\
Total DFS score & $0.596^{* *}$ & $0.314^{*}$ & 0.221 & 236 & 228 \\
Saturated fat scale & $0.293^{*}$ & -0.224 & -0.131 & $0.602^{* *}$ & $0.319^{* *}$ \\
Free sugar scale & $0.473^{* *}$ & 0.142 & $0.302^{*}$ & $0.359^{* *}$ & $0.504^{* *}$ \\
\hline
\end{tabular}

* Correlation is significant at 0.05 level (two-tailed). ** Correlation is significant at 0.01 level.

Table 3. Quartiles of the DFS scores, range, sample size, and mean values of energy intake and the percentage of energy from saturated fat and free sugar

\begin{tabular}{rllllll}
\hline Quartile & $\begin{array}{l}\text { Range total } \\
\text { DFS score }\end{array}$ & $n$ & $\begin{array}{l}\text { Total energy } \\
\text { intake, kJ }\end{array}$ & $\begin{array}{l}\text { Saturated } \\
\text { fat }\end{array}$ & $\begin{array}{l}\text { Free } \\
\text { sugar }\end{array}$ & $\begin{array}{l}\text { Free } \\
\text { sucrose }\end{array}$ \\
\hline $25 \%$ & $32-45$ & 16 & 170,107 & $14.88 \%$ & $12.70 \%$ & $4.26 \%$ \\
$50 \%$ & $46-53$ & 14 & 215,664 & $15.22 \%$ & $16.98 \%$ & $7.22 \%$ \\
$75 \%$ & $54-60$ & 18 & 211,249 & $17.08 \%$ & $20.08 \%$ & $8.22 \%$ \\
$100 \%$ & $61-91$ & 17 & 311,430 & $16.95 \%$ & $21.91 \%$ & $8.97 \%$ \\
\hline
\end{tabular}

There was no significant gender difference in the total DFS score $(t(63)=-1.302, p=$ 0.198). Also, no significant gender differences in energy intake, free sugar, and saturated fat intake derived from the FFQ were found. The DFS score did not significantly correlate with BMI $(r=-0.129, p=0.307)$ or age $(r=-0.167, p=0.183)$. Correlations of the total DFS score, the fat, sugar, and fat-sugar subscales with total energy and target nutrient intake derived from the FFQ are reported in Table 2.

Significant negative correlations of the total score were found for intrinsic fructose, glucose, and sucrose ( $r=-0.343$ to $-0.357, p \leq 0.01$ ). Of non-target nutrients, only monosaturated fatty acids $(r=0.358, p=0.003)$ and protein $(r=0.401, p \leq 0.001)$ correlated positively with the fat subscale. No other non-target nutrients were positively correlated with any subscale or the total score.

Average nutrient intakes of the subjects separated into quartiles according to the DFS score are reported in Table 3. Subjects in the upper quartile had significant higher nutrient intakes of free sugar in the form of carbohydrates $(Z=-3.17, p \leq 0.001)$ and sucrose $(Z=$ $-3.314, p \leq 0.001)$. No significant difference between the quartiles was found for fat and satu- 
rated fatty acids. The DFS score was significantly correlated with restraint $(r=-0.380, p=$ $0.002)$ and hunger $(r=0.275, p=0.026)$. Correlations between the DFS score and disinhibition, BAS, and BAS-RR failed to be significant.

By means of regression analyses, we investigated whether the relationship between nutrient estimates and DFS scores was moderated by BMI. Moderation of the relationship between free sugar in the form of sucrose or carbohydrates and the total DFS failed to be significant. However, we found a significant moderation of DFS scores and saturated fat by BMI $\left(R^{2}=0.156, F(3,61)=3.757, p=0.015\right)$ as indicated by a significant interaction between BMI and DFS scores on saturated fat intake $(\beta=-0.0001, p=0.048)$. Examination of the conditional effects indicated that regression coefficients of DFS for saturated fat decreased with BMI.

\section{Discussion}

The present study provides first empirical support for the psychometric properties of the German version of the DFS. Test-retest reliability (intraclass correlation coefficient $=0.801$ ), internal consistency of the total DFS score $(\alpha=0.808)$, and subscales $(\alpha=0.513-0.703)$ are comparable to the original English version [13] as well as to other short questionnaires [36] and screening tools assessing dietary fat intake [37]. Subscales listing food that contains predominantly saturated fat, free sugar, or saturated fat and free sugar combined correlated with the total DFS score, further indicating internal consistency. Validity was assessed relative to a comprehensive FFQ (DEGS-Ernährungsfragebogen). We found positive correlations of the total DFS score with self-reported saturated fat intake $(r=0.258)$, intake of free sugar $(r=$ $0.443)$, and sucrose $(r=0.445)$. Discriminant validity of the total DFS score was supported as it was not correlated with protein, mono- and polyunsaturated fatty acids, or intrinsic sugar. Intrinsic sugar was significantly negatively correlated with DFS scores, which is in line with prior research [38] indicating that people who eat lots of free sugar choose less food rich in intrinsic sugar, like fruits. Suggesting high convergent and discriminant validity, the DFS subscales that are specific for saturated fat and free sugar were correlated only with the nutrients they intended to measure.

Similar to the English version, no correlation of the total DFS score with BMI was found [13]. Although one would expect individuals with obesity to have a higher intake of food listed in the DFS, this is in line with previous research showing that obese people restrain their fat and sugar intake with the intention to lose weight $[14,39]$. Alternatively, the results could reflect weight group-specific underreporting which was found to be more pronounced in individuals with overweight and obesity [40]. Underreporting is also a plausible reason for the finding that regression coefficients between the DFS score and saturated fat intake decreased with increasing BMI. This indicates that the DFS represents saturated fat intake of individuals with a BMI $>30$ not as precisely as of individuals with a normal-weight BMI. The strength of association between DFS and free sugar estimates was not moderated by BMI, indicating that the DFS score is an equally valid indicator for free sugar intake of individuals across weight groups. Lastly, since underreporting is specifically observed for food considered as unhealthy, particularly fat [41], and because the DFS explicitly inquires intake of food considered as unhealthy, underreporting may be greater in the DFS than the comprehensive FFQ which inquires all sorts of foods. Consequently, correlations between the FFQ and DFS, especially for fat intake, might have been underestimated. It is for future research to examine to what extent DFS scores are biased by social desirability effects and to evaluate the validity for different weight groups with larger sample sizes.

We expected men to score higher on the DFS than women, in accordance with previous research [42]. However, no such gender differences were found. Considering that total energy 
and target nutrient intake, measured with the FFQ, did not differ between men and women either, this must not be considered as impairment of the DFS, but reflects a true lack of difference of reported dietary intake in this sample. There was no significant correlation with age, possibly due to the narrow age range in the sample. We compared individuals scoring in the lower and upper quartile of the DFS with respect to their self-reported nutrient intake from the FFQ. Total energy and free sugar intake was higher for individuals in the upper DFS quartile. Although pointing towards the hypothesized direction, no significant differences were found for total fat and saturated fat. In line with previous findings [14-16], DFS scores increased with self-reported feelings of hunger and decreased with eating restraint. DFS scores and disinhibition were not associated. As disinhibition and sensitivity to reward are closely intertwined [43], it relates to the lack of association between self-reported sensitivity to reward and DFS scores. This may be interpreted as failure of the DFS to replicate previous findings or as invalidity of our hypothesis that higher saturated fat and free sugar intake is associated with higher sensitivity to reward. Some argue that high fat and sugar intake may be associated with lower reward sensitivity due to the development of tolerance [44]. Consequently, more sugar and fat are needed to achieve accustomed experiences of reward from food consumption, consequently increasing intake to compensate for this surfeit. Research suggests that the direction of the relation between reward sensitivity and dietary intake might depend on weight status and dietary habits $[45,46]$. Thus, relating reward sensitivity to intake of fatty and sugary foods is far more complex than we assumed in our hypothesis, hampering the interpretation of these results.

Several limitations of the DFS format should be kept in mind when interpreting the score. First, high scores might reflect high food intake overall and not a high proportion of saturated fat and free sugar in the diet. Calorie requirements depend on gender, body size, metabolic circumstances, daily activity, and exercise levels. Individuals with higher calorie demand might eat food listed in the DFS more often just because they eat more in total. Second, saturated fat and free sugar intake is calculated as the product of eating frequency and amount. The DFS only assesses frequency and fails to take portion size into account. Third, the authors of the original DFS did not derive the subscales factor analytically. They based item assignment to subscales on evaluation of nutrient content, thus leaving the factor structure of the subscales questionable. A recognized limitation of our study is the small sample size and that the sample is not representative of the general population but selected to contain similar numbers of individuals with normal weight, overweight, and obesity. Therefore, generalizability to the German population is limited, and future research should examine psychometrics of the DFS in a larger sample. Another obstacle of self-report instruments like the DFS and FFQ, which we used here, is that they suffer from memory bias and individual answer tendency. This may inflate their agreement without measuring actual dietary intake. On the other hand, the FFQ assesses food consumption in the past 4 weeks, whereas the DFS takes the past 12 months into account, possibly decreasing the association between the scores of the two questionnaires. However, to our knowledge no comprehensive FFQ that asks about 4 months was available. Future research should compare the validity of the DFS with that of instruments with different error structure, such as food records or repeated 24-h recalls [20,47].

Our findings corroborate the usability of the DFS as a screening tool to identify people with high/low saturated fat and free sugar intake. Francis and Stevenson [13] suggest that persons with scores $>60$, which marked the median in their sample, should be advised to lower their intake. Although in our sample a score $>60$ marks the upper quartile, we suggest keeping this criterion as a slightly more conservative cut-off for the German population. Potential applications of the DFS in research are the identification of samples with a high/low saturated fat and free sugar intake. In clinical practice, it provides a quick overview of dietary habits or might be used to control treatment success in dietary interventions. 


\section{Conclusion}

Our results suggest that the German version of the DFS might be a psychometrically sound instrument to identify individuals with particularly high or low intake of saturated fat and free sugar. Although further testing in larger samples is recommended, the DFS seems to be equally valid and reliable to assess free sugar intake in different weight groups. Our data suggest that assessment of saturated fat intake may be somewhat less valid for individuals with obesity. The DFS is quick to administer, score, and interpret for professionals and patients. Therefore, it may be a valuable self-rating questionnaire for researchers and clinicians in Germany.

\section{Acknowledgements}

The authors thank Laura Rehberger for her perceptive thoughts and her contribution to the setup and conduct of the study. Translation: Nora Mehl, Suse Prejawa. Assisting in logistics and digitalization of questionnaires: Suse Prejawa. Consulting regarding FFQ and Nutrition software: Sebastian Huhn, Tatjana Schütz. Thoughtful comments on previous version of manuscript: Lieneke K. Janssen and an anonymous reviewer.

\section{Statement of Ethics}

This study was conducted according to the standards laid down in the Declaration of Helsinki. All procedures were approved by the MPI/CBS Ethics Committee. The study is registered at the Max Planck Institute for Human Cognitive and Brain Science with the identifier "NRO-170 VALDFS." All participants provided signed consent to participate in the study.

\section{Disclosure Statement}

The authors declare that they have no competing interests.

\section{Funding Sources}

This work was supported by grants from the Federal Ministry of Education and Research (BMBF), Germany (to A.H.; FKZ: 01E01001), and the Deutsche Forschungsgemeinschaft (DFG, German Research Foundation) to A.H.; Projektnummer 209933838 - SFB 1052, subproject A5.

\section{Author Contributions}

This research was conducted in collaboration with the Department Neurology, Max Planck Institute for Human Cognitive and Brain Sciences, in the O'BRAIN Lab under advice of AH. Planning, preparation of the study, and analysis of the results was done collaboratively. S.P.F. guided the participants and wrote the manuscript for the present paper. A.H. provided supervision throughout the study and feedback on the manuscript. Both authors approved the final version of the manuscript. 
Fromm and Horstmann: German Dietary Fat and Free Sugar-Short Questionnaire

\section{References}

1 World Health Organization. Global status report on noncommunicable diseases 2014. Geneva: WHO; 2014. p. 302.

2 World Health Organization. Sugars intake for adults and children. Guideline. Geneva: WHO; 2015. p. 49.

3 DiNicolantonio JJ, Lucan SC, O'Keefe JH. The Evidence for Saturated Fat and for Sugar Related to Coronary Heart Disease. Prog Cardiovasc Dis. 2016 Mar-Apr;58(5):464-72.

4 Malik VS, Popkin BM, Bray GA, Després JP, Willett WC, Hu FB. Sugar-sweetened beverages and risk of metabolic syndrome and type 2 diabetes: a meta-analysis. Diabetes Care. 2010 Nov;33(11):2477-83.

5 Chen L, Caballero B, Mitchell DC, Loria C, Lin PH, Champagne CM, et al. Reducing consumption of sugarsweetened beverages is associated with reduced blood pressure: a prospective study among United States adults. Circulation. 2010 Jun;121(22):2398-406.

6 Malik VS, Pan A, Willett WC, Hu FB. Sugar-sweetened beverages and weight gain in children and adults: a systematic review and meta-analysis. Am J Clin Nutr. 2013 Oct;98(4):1084-102.

7 Hu FB. Resolved: there is sufficient scientific evidence that decreasing sugar-sweetened beverage consumption will reduce the prevalence of obesity and obesity-related diseases. Obes Rev. 2013 Aug;14(8):606-19.

8 Te Morenga L, Mallard S, Mann J. Dietary sugars and body weight: systematic review and meta-analyses of randomised controlled trials and cohort studies. BMJ. 2012 Jan 15;346:e7492.

9 Natarajan P, Ray KK, Cannon CP. High-density lipoprotein and coronary heart disease: current and future therapies. J Am Coll Cardiol. 2010 Mar;55(13):1283-99.

10 Astrup A, Dyerberg J, Elwood P, Hermansen K, Hu FB, Jakobsen MU, et al. The role of reducing intakes of saturated fat in the prevention of cardiovascular disease: where does the evidence stand in 2010 ? Am J Clin Nutr. 2011 Apr;93(4):684-8.

11 Ernst JB, Arens-Azevedo U, Bitzer B, Bosy-Westphal A, de Zwaan M, Egert S, et al. Quantitative recommendation on sugar intake in Germany. Short version of the consensus paper by the German Obesity Society (DAG), German Diabetes Society (DDG) and German Nutrition Soeciety (DGE). Ernahr-Umsch. 2019;66(2): 26-34.

12 Deutsche Gesellschaft für Ernährung e.V. Evidenzbasierte Leitlinie: Fettzufuhr und Prävention ausgewählter ernährungsmitbedingter Krankheiten. Bonn: Deutsche Gesellschaft für Ernährung; 2015. p. 1-231.

13 Francis H, Stevenson R. Validity and test-retest reliability of a short dietary questionnaire to assess intake of saturated fat and free sugars: a preliminary study. J Hum Nutr Diet. 2013 Jun;26(3):234-42.

14 Keskitalo K, Tuorila H, Spector TD, Cherkas LF, Knaapila A, Kaprio J, et al. The Three-Factor Eating Questionnaire, body mass index, and responses to sweet and salty fatty foods: a twin study of genetic and environmental associations. Am J Clin Nutr. 2008 Aug;88(2):263-71.

15 Contento IR, Zybert P, Williams SS. Relationship of cognitive restraint of eating and disinhibition to the quality of food choices of Latina women and their young children. Prev Med. 2005 Mar;40(3):326-36.

16 Lähteenmäki L, Tuorila H. Three-factor eating questionnaire and the use and liking of sweet and fat among dieters. Physiol Behav. 1995 Jan;57(1):81-8.

17 De Cock N, Van Lippevelde W, Vervoort L, Vangeel J, Maes L, Eggermont S, et al. Sensitivity to reward is associatd with snack and sugar-sweetened beverage consumption in adolescents. Eur J Nutr. 2016;55(4):1623-32.

18 Davis C, Patte K, Levitan R, Reid C, Tweed S, Curtis C. From motivation to behaviour: a model of reward sensitivity, overeating, and food preferences in the risk profile for obesity. Appetite. 2007 Jan;48(1):12-9.

19 Haftenberger M, Heuer T, Heidemann C, Kube F, Krems C, Mensink GB. Relative validation of a food frequency questionnaire for national health and nutrition monitoring. Nutr J. 2010 Sep;9(1):36.

20 Rohrmann S, Klein G. Validation of a short questionnaire to qualitatively assess the intake of total fat, saturated, monounsaturated, polyunsaturated fatty acids, and cholesterol. J Hum Nutr Diet. 2003 Apr;16(2): 111-7.

21 Mensink GB, Haftenberger M, Thamm M. Validity of DISHES 98, a computerised dietary history interview: energy and macronutrient intake. Eur J Clin Nutr. 2001 Jun;55(6):409-17.

22 Beck AT, Ward CH, Mendelson M, Mock J, Erbaugh J. An inventory for measuring depression. Arch Gen Psychiatry. 1961 Jun;4(6):561-71.

23 Aardoom JJ, Dingemans AE, Slof Op't Landt MC, Van Furth EF. Norms and discriminative validity of the Eating Disorder Examination Questionnaire (EDE-Q). Eat Behav. 2012 Dec;13(4):305-9.

24 Brewin N, Baggott J, Dugard P, Arcelus J. Clinical normative data for eating disorder examination questionnaire and eating disorder inventory for DSM-5 feeding and eating disorder classifications: a retrospective study of patients formerly diagnosed via DSM-IV. Eur Eat Disord Rev. 2014 Jul;22(4):299-305.

25 Schmitz C. LimeSurvey: An open source survey tool. Hamburg: LimeSurvey Project Hamburg; 2012.

26 Stunkard AJ, Messick S. The three-factor eating questionnaire to measure dietary restraint, disinhibition and hunger. J Psychosom Res. 1985;29(1):71-83.

27 Carver CS, White TL. Behavioral Inhibition, Behavioral Activation, and Affective Responses to Impending Reward and Punishment: the BIS/BAS Scales. J Pers Soc Psychol. 1994;67(2):319-33.

28 Fairburn CG, Cooper PJ. The Eating Disorder Examination. In: Fairburn CG, Wilson GT, editors. Binge eating Nature, assessment, and treatment. 12th ed. New York: Guilford Press; 1993. pp. 317-60.

29 Strobel A, Beauducel A, Debener S, Brocke B. Eine deutschsprachige Version des BIS/BAS-Fragebogens von Carver und White. Zeitschrift für Differ und Diagnostische Psychol. 2001 Sep;22(3):216-27. 
Fromm and Horstmann: German Dietary Fat and Free Sugar-Short Questionnaire

30 Müller A, Smits D, Claes L, de Zwaan M. Faktorenstruktur der deutschsprachigen Version der BIS/BAS-Skalen in einer Bevölkerungsstichprobe. Fortschr Neurol Psychiatr. 2013;81(2):75-80.

31 Pudel V, Westenhöfer J. Fragebogen zum Eßverhalten. (FEV); Handanweisung. Göttingen: Verl. für Psychologie Hogrefe; 1989.

32 Löffler A, Luck T, Then FS, Sikorski C, Kovacs P, Böttcher Y, et al. Eating Behaviour in the General Population: An Analysis of the Factor Structure of the German Version of the Three-Factor-Eating-Questionnaire (TFEQ) and Its Association with the Body Mass Index. PLoS One. 2015 Jul;10(7):e0133977.

33 Hilbert A, Tuschen-Caffier B, Karwautz A, Niederhofer H, Munsch S. Eating Disorder Examination-Questionnaire. Diagnostica. 2007;53(3):144-54.

34 Hautzinger M, Bailer M, Worall H, Keller F. BDI Beck-Depressions-Inventar Testhandbuch. Bern: Hans Huber. Bern: Hans Huber; 1995.

35 Bernstein JT, Schermel A, Mills CM, L'Abbé MR. Total and Free Sugar Content of Canadian Prepackaged Foods and Beverages. Nutrients. 2016 Sep;8(9):E582.

36 England CY, Andrews RC, Jago R, Thompson JL. A systematic review of brief dietary questionnaires suitable for clinical use in the prevention and management of obesity, cardiovascular disease and type 2 diabetes. Eur J Clin Nutr. 2015 Sep;69(9):977-1003.

37 Calfas KJ, Zabinski MF, Rupp J. Practical nutrition assessment in primary care settings: a review. Am J Prev Med. 2000 May;18(4):289-99.

38 Louie JC, Tapsell LC. Association between intake of total vs added sugar on diet quality: a systematic review. Nutr Rev. 2015 Dec;73(12):837-57.

39 Anglé S, Engblom J, Eriksson T, Kautiainen S, Saha MT, Lindfors P, et al. Three factor eating questionnaire-R18 as a measure of cognitive restraint, uncontrolled eating and emotional eating in a sample of young Finnish females. Int J Behav Nutr Phys Act. 2009 Jul;6(1):41.

40 Braam LA, Ocké MC, Bueno-de-Mesquita HB, Seidell JC. Determinants of obesity-related underreporting of energy intake. Am J Epidemiol. 1998 Jun;147(11):1081-6.

41 Goris AH, Westerterp-Plantenga MS, Westerterp KR. Undereating and underrecording of habitual food intake in obese men: selective underreporting of fat intake. Am J Clin Nutr. 2000 Jan;71(1):130-4.

42 Heuer T, Krems C, Moon K, Brombach C, Hoffmann I. Food consumption of adults in Germany: results of the German National Nutrition Survey II based on diet history interviews. Br J Nutr. 2015 May;113(10):1603-14.

43 Mobbs 0, Crépin C, Thiéry C, Golay A, Van der Linden M. Obesity and the four facets of impulsivity. Patient Educ Couns. 2010 Jun;79(3):372-7.

44 Davis C, Strachan S, Berkson M. Sensitivity to reward: implications for overeating and overweight. Appetite. 2004 Apr;42(2):131-8.

45 Burger KS, Stice E. Variability in reward responsivity and obesity: evidence from brain imaging studies. Curr Drug Abuse Rev. 2011 Sep;4(3):182-9.

46 Burger KS, Stice E. Elevated energy intake is correlated with hyperresponsivity in attentional, gustatory, and reward brain regions while anticipating palatable food receipt. Am J Clin Nutr. 2013 Jun; 97(6):1188-94.

47 Cade J, Thompson R, Burley V, Warm D. Development, validation and utilisation of food-frequency questionnaires - a review. Public Health Nutr. 2002 Aug;5(4):567-87. 